OPEN ACCESS

Edited by:

Vincent Kam Wai Wong,

Macau University of Science and

Technology, Macau

Reviewed by:

Yun Zhang,

University of Macau, Macau Yih-Fung Chen,

Kaohsiung Medical University, Taiwan

*Correspondence:

Jiansheng $L i$

lijs8@163.com

${ }^{t}$ These authors have contributed equally to this work

Specialty section:

This article was submitted to Ethnopharmacology,

a section of the journal

Frontiers in Pharmacology

Received: 01 April 2020

Accepted: 24 July 2020

Published: 07 August 2020

Citation:

Zhao P, Liu X, Dong H, Tian Y, Feng S,

Zhao $D$, Ren Z, Zhang L and Li J (2020) Bufei Yishen Formula Restores

Th17/Treg Balance and Attenuates

Chronic Obstructive Pulmonary

Disease via Activation of the

Adenosine 2a Receptor.

Front. Pharmacol. 11:1212.

doi: 10.3389/fphar.2020.01212

\section{Bufei Yishen Formula Restores Th17/ Treg Balance and Attenuates Chronic Obstructive Pulmonary Disease via Activation of the Adenosine 2a Receptor}

\author{
Peng Zhao ${ }^{1,2,3 \dagger}$, Xuefang Liu ${ }^{1,2,3 \dagger}$, Haoran Dong ${ }^{1,2,3}$, Yange Tian ${ }^{1,2,3}$, Suxiang Feng ${ }^{1,2}$, \\ Di Zhao ${ }^{1,2,3}$, Zhouxin Ren ${ }^{1,2,3}$, Lanxi Zhang ${ }^{1,2,3}$ and Jiansheng $\mathrm{Li}^{1,2,3 *}$ \\ ${ }^{1}$ Co-construction Collaborative Innovation Center for Chinese Medicine and Respiratory Diseases by Henan \& Education \\ Ministry of P.R. China, Henan University of Chinese Medicine, Zhengzhou, China, ${ }^{2}$ Henan Key Laboratory of Chinese \\ Medicine for Respiratory Disease, Henan University of Chinese Medicine, Zhengzhou, China, ${ }^{3}$ Academy of Chinese Medical \\ Sciences, Henan University of Chinese Medicine, Zhengzhou, China
}

Bufei Yishen formula (BYF) is a Traditional Chinese Medicine (TCM) reported to ameliorate chronic obstructive pulmonary disease (COPD) by regulating the balance between $T$ helper (Th) 17 and regulatory $T$ (Treg) cells. However, its mechanism remains unknown. Therefore, this study aimed to explore the underlying mechanisms of BYF. Naïve CD4+ $T$ cells were exposed to anti-CD3, anti-CD28, transforming growth factor (TGF)- $\beta$, and/or interleukin (IL)-6 to promote their differentiation into Th17 or Treg cells. A rat model of cigarette smoke- and bacterial infection-induced COPD was established and orally treated with BYF and/or an adenosine $2 \mathrm{a}$ receptor (A2aR) antagonist. Then, the rats were sacrificed, their lung tissues were removed for histological analysis, and their spleens were collected to evaluate Th17 and Treg cells. The results showed that BYF significantly suppressed Th17 cell differentiation and its related cytokines and enhanced Treg cell differentiation and its related cytokines. In addition, BYF activated the A2aR, increased the levels of p-signal transducer and activator of transcription (STAT)5, and decreased the level of p-STAT3 in Treg and Th17 cells. The A2aR antagonist suppressed the changes induced by BYF treatment in Th17 and Treg cells. Furthermore, the A2aR antagonist diminished the therapeutic effect of BYF on COPD, as indicated by the lung injury scores, bronchiole wall thickness, small pulmonary vessels wall thickness, bronchiole stenosis, alveolar diameters, decrease in inflammatory cytokines, increase in alveolar number, and lung functions. Similarly, the A2aR antagonist reversed the effects of BYF on the proportion of Th17 and Treg cells in the spleen. Additionally, BYF increased the protein and mRNA levels of A2aR and regulated the phosphorylation of STAT3 and STAT5 in spleen and lung tissues, which were inhibited by cotreatment with the A2aR antagonist. In conclusion, this study suggested that BYF exhibited its anti-COPD efficacy by restoring 
the Th17/Treg balance via activating A2aR, which may provide evidence for the clinical application of BYF in the treatment of COPD.

Keywords: Bufei Yishen formula, chronic obstructive pulmonary disease, adenosine 2a receptor, Th17 cell, Treg cell, Th17/Treg balance

\section{INTRODUCTION}

Chronic obstructive pulmonary disease (COPD) is a heterogeneous syndrome associated with abnormal inflammatory immune responses of the lung. COPD is a worldwide public health challenge because of its high prevalence and related disability and mortality (Wang et al., 2018; Maigeng et al., 2019). Although the precise pathogenesis of COPD remains unclear, Th17/Treg imbalance is considered to be an essential inducer of COPD development (Barnes, 2016). In COPD patients, the Th17/Treg cell balance shifts toward Th17 cells, which triggers inflammatory responses in the airways and lungs and exacerbates alveolar destruction by producing interleukin-17 (IL-17) (Brusselle et al., 2011; Eppert et al., 2013). For instance, Th17 expansion and higher IL-17A concentrations are found in the sputum of COPD patients and are tightly correlated with increased neutrophil chemotactic mediators and airway obstruction (Le Rouzic et al., 2017; Cervilha et al., 2019). In vitro, Th17 cells can be generated from naïve T-cells induced by transforming growth factor (TGF)- $\beta 1$ and IL-6. Additionally, IL-17A produced by Th17 cells is tightly regulated by transcriptional factors, including signal transducer and activator of transcription 3 (STAT3) and retinoid-related orphan receptor (ROR) $\gamma t$, which are activated by combinations of cytokines, including IL-6 and TGF- $\beta$ (Jin et al., 2014; Cervilha et al., 2019). In contrast to Th17 cells, Treg cells have immunoregulatory functions and secrete anti-inflammatory cytokines to strengthen immune tolerance and inhibit inflammatory responses. Treg levels are decreased in COPD patients and mice induced by chronic cigarette smoke exposure (Yang et al., 2011; Zhang et al., 2016). For example, the number of Tregs in the small airways and peripheral blood of COPD patients is lower compared with healthy individuals. In addition, Treg cells in COPD patients exhibit a lower capacity to inhibit inflammation (Jin et al., 2014; Sales et al., 2017). Therefore, the Th17 and Treg balance is essential to maintain immune homeostasis and inhibit inflammation in COPD.

The adenosine $2 \mathrm{a}$ receptor $(\mathrm{A} 2 \mathrm{aR})$ is widely expressed on $\mathrm{T}$ cell subsets and may regulate cytokine production in activated $\mathrm{T}$ lymphocytes, suggesting that this receptor may play a role in immune homeostasis and inflammatory processes (Zarek et al., 2008; Li N. et al., 2012). A2aR stimulation promotes the generation of forkhead box $\mathrm{p} 3$ (Foxp3)+ regulatory $\mathrm{T}$ cells and reduces Th17-cell subtypes (Zarek et al., 2008). Additionally, A2aR-knockout mice sensitized and challenged with ragweed exhibit enhanced airway inflammation and hyperresponsiveness compared with wild-type mice (Haskó et al., 2008). Further, A2aR agonist treatment inhibits airway inflammation in allergen-sensitized and -challenged brown Norway rats (Bonneau et al., 2006; Trevethick et al., 2008). In addition, A2aR signaling pathways activate protein kinase A (PKA), resulting in CRE binding protein (CREB) phosphorylation, and A2aR silencing on $\mathrm{T}$ cells can block their differentiation toward Tregs by inhibiting PKA/CREB activation (Yang et al., 2015; Masjedi et al., 2019). Thus, activating A2aR to restore the Th17/ Treg balance may offer a potential therapeutic strategy for COPD.

Bufei Yishen formula (BYF) (patent: ZL.201110117578.1) is a traditional Chinese formula composed of twelve Chinese medicines, including Ginseng Radix et Rhizoma, Astragali Radix, Corni Fructus, Lycii Fructus, Schisandrae Chinensis Fructus, Fritillariae Thunbergii Bulbus, Perillae Fructus, Citri Reticulatae Pericarpium, Epimedii Folium, Paeoniae Rubra Radix, Pheretima, and Ardisiae Japonicae Herba. Previous clinical studies reported the beneficial effects of BYF on measured outcomes in stable COPD patients over the 6-month treatment and 12-month follow-up periods. Specifically, BYF alleviated COPD symptoms by reducing the exacerbation frequency, delaying acute exacerbation, and improving pulmonary function and exercise capacity (Li S. et al., 2012). We previously conducted a system analysis by integrating transcriptomics, proteomics, metabolomics, and system pharmacology and showed that BYF achieved its beneficial effect on COPD by regulating immune responses, inflammatory responses, lipid metabolism, and other processes (Li et al., 2015; Li J. et al., 2016). Subsequently, we demonstrated that BYF restored the Th17/Treg balance in spleens and mesenteric lymph nodes and modulated the activities of STAT3 and STAT5 in COPD rats (P et al., 2018). However, the detailed mechanisms are still unknown and require further investigation.

The present work aimed to explore the mechanism by which BYF restores the Th17/Treg balance in vitro and in vivo and evaluate the critical role of $\mathrm{A} 2 \mathrm{aR}$ in the subsequent anti-COPD effect of BYF.

\section{MATERIALS AND METHODS}

\section{Chemicals and Reagents}

Tobacco was purchased from Henan Tobacco Industry (Hongqi Canal ${ }^{\circledR}$ Filter tip cigarette; tobacco type, tar: $10 \mathrm{mg}$; nicotine content: $1.0 \mathrm{mg}$; carbon monoxide: $12 \mathrm{mg}$, Zhengzhou, China). Klebsiella pneumoniae (strain ID: 46114) was obtained from the National Center for Medical Culture Collection (CMCC, Beijing, China). Aminophylline was purchased from Shandong Xinhua Pharmaceutical Co., LTD. (Shandong, China). KW6002 was obtained from MedChem Express (Shanghai, China). AntiCD4, IL-17A, CD25, and Foxp3 antibodies were obtained from eBioscience, Inc. (Affymetrix, CA, USA). Rat IL-1 $\beta$, tumor 
necrosis factor (TNF)- $\alpha$, IL-6, IL-17A, and IL-10, ELISA kits were purchased from Boster Biological Engineering (Wuhan, China). TGF- $\beta$, IL-6, and anti-CD3/CD28 antibodies were obtained from BD Biosciences (Franklin Lakes, USA). The mouse $\mathrm{CD}^{+} \mathrm{CD}^{2} \mathrm{~L}^{+} \mathrm{T}$ Cell Isolation Kit was obtained from Miltenyi Biotec Inc. (Miltenyi, CA, USA). Anti-A2aR, Foxp3, ROR $\gamma$, p-STAT3 (Tyr705), p-STAT5 (Tyr694), STAT3, and STAT5 antibodies were purchased from Santa Cruz Biotechnology (Santa Cruz, CA, USA). HiScript QRTSuperMix and AceQ quantitative polymerase chain reaction (qPCR) SYBR Green Master Mix were purchased from Vazyme Biotech (Nanjing, China).

\section{Animals}

Sixty-two Sprague-Dawley rats $(200 \pm 20 \mathrm{~g})$ and $20 \mathrm{C} 57 \mathrm{BL} / 6$ mice were obtained from SPF biotechnology co., LTD. (Beijing, China). Animals were maintained in specific pathogen-free facilities and housed in filter-top cages with free access to food and water under a 12-h light: 12 -h dark cycle in plastic cages at $25^{\circ} \mathrm{C} \pm 2{ }^{\circ} \mathrm{C}$ with a relative humidity of $50 \% \pm 10 \%$. The animal experiments were approved by the Experimental Animal Care and Ethics Committee of the First Affiliated Hospital, Henan University of Chinese Medicine.

\section{BYF Preparation}

BYF is composed of 12 Chinese medicines (Table 1). These Chinese medicines were identified by professor Suxiang Feng and then prepared as dry extracts as described previously (Li Y. et al., 2014). High-performance liquid chromatography (HPLC) of BYF was performed, and 10 chemical constituents of BYF were identified according to the spectrograms and retention times of their standard substances using HPLC-UV (254 nm) (Figure 1). Finally, the per gram dry extract obtained was equivalent to $3.79 \mathrm{~g}$ raw medical herbs. This dry extract was given orally to COPD rats.

The BYF dry extract was dissolved in double-distilled water at a $0.511 \mathrm{~g} / \mathrm{ml}$ concentration. Then, the $70 \%$ ethanol extract was loaded on a DM101 macroporous resin column, and the $95 \%$ aqueous ethanol elution fraction was collected. Following the collection, the extracts were concentrated to total dryness by vacuum freezedrying, and $1.6984 \mathrm{~g}$ of powders were acquired. Finally, the pergram dry extract obtained was equivalent to $30 \mathrm{~g}$ raw medical herbs. This dry extract was prepared for cellular experiments.

\section{Naïve CD4 ${ }^{+} \mathrm{T}$ Cell Isolation and Differentiation}

Naïve CD4+ T cells from mouse spleens were isolated with magnetic beads $(\mathrm{CD} 4+\mathrm{CD} 62 \mathrm{~L}+\mathrm{T}$ Cell Isolation Kit II, Miltenyi

TABLE 1 | The compositions of Bufei Yishen formula.

\begin{tabular}{|c|c|c|c|c|}
\hline No. & Herbal drug & Latin scientific name & plant part(s) & Amount (g) \\
\hline 1 & Ginseng Radix et Rhizoma & Panax ginseng C.A. Mey & Radix et Rhizoma & 9 \\
\hline 2 & Astragali Radix & Astragalus tibetanus Bunge & Radix & 15 \\
\hline 3 & Corni Fructus & Cornus officinalis Siebold \& Zucc & Fructus & 12 \\
\hline 4 & Lycii Fructus & Lycium barbarum L. & Fructus & 12 \\
\hline 5 & Schisandrae Chinensis Fructus & Schisandra arisanensis Hayata & Fructus & 9 \\
\hline 6 & Fritillariae Thunbergii Bulbus & Fritillaria thunbergii Miq & Bulbus & 9 \\
\hline 7 & Perillae Fructus & Perilla frutescens (L.) Britton & Fructus & 9 \\
\hline 8 & Citri Reticulatae Pericarpium & Citrus sinensis (L.) Osbeck & Pericarpium & 9 \\
\hline 9 & Epimedii Folium & Epimedium acuminatum Franch & Folium & 9 \\
\hline 10 & Paeoniae Rubra Radix & Paeonia anomala L & Radix & 9 \\
\hline 11 & Pheretima & Pheretima aspergillum (E. Perrier) & & 12 \\
\hline 12 & Ardisiae Japonicae Herba & Ardisia japonica (Thumb.) Blume & Herba & 15 \\
\hline
\end{tabular}

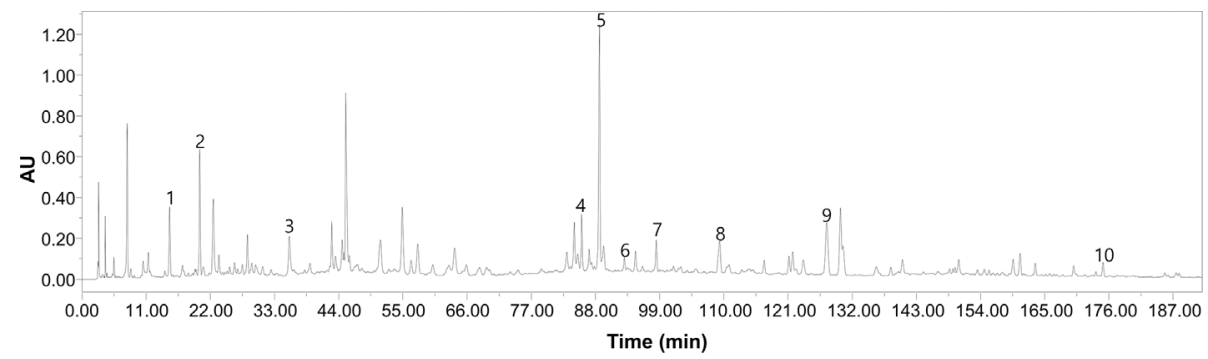

FIGURE 1 | The high-performance liquid chromatography (HPLC)-UV chromatogram of Bufei Yishen Formula (BYF). Peak number and identity, 1: ursolic acid (PubChem CID: 64945); 2: bergenin (PubChem CID: 66065); 3: paeoniflorin (PubChem CID: 442534); 4: Epimedin C (PubChem CID:5748394); 5: icariin (PubChem CID:5318997); 6: Kaempferol (PubChem CID: 5280863); 7: deoxyschizandrin (PubChem CID:155256); 8: ginsenoside Rd (PubChem CID: 24721561); 9: Schisandrin (PubChem CID:23915); 10: Schisandrin B (PubChem CID:108130). 
Biotech). TGF $\beta$ is essential for Th17 and Treg differentiation and activates both Foxp 3 and ROR $\gamma$ t expression. However, TGF $\beta$ can exclusively induce $\mathrm{T}$ cell differentiation into Tregs, in which higher levels of Foxp3 inhibit ROR $\gamma$ t transcriptional activation. In the presence of both TGF- $\beta$ and IL- 6 , this inhibition of ROR $\gamma$ t was abrogated, and Th17 differentiation was initiated by activating STAT3 and other distinct transcriptional programs (Korn et al., 2009; Kimura and Kishimoto, 2010). Naïve CD4+ T cells were cultured in vitro with anti-CD3 and anti-CD28 (2 $\mu \mathrm{g} /$ $\mathrm{ml}$ ) under Th17 (TGF- $\beta$, $5 \mathrm{ng} / \mathrm{ml}$; IL-6, 20 ng/ml) or Treg (TGF$\beta, 5 \mathrm{ng} / \mathrm{ml}$ ) differentiation conditions in the presence/absence of BYF $(60 \mu \mathrm{g} / \mathrm{ml})$ or KW6002 $(0.1 \mu \mathrm{M})$. After stimulation for 3 days, the cells were harvested for analysis.

\section{COPD Rat Model and Drug Administration}

COPD rat preparation and drug administration were described previously (Li Y. et al., 2012). Briefly, rats were maintained in a closed chamber exposed to tobacco from weeks 1-12 and repeated $K$. pneumoniae infections from weeks $1-8$. The COPD rats were orally treated with normal saline, BYF (4.4 g/ $\mathrm{kg})$, KW6002 (2 mg/kg), BYF (4.4 g/kg) + KW6002 (2 mg/kg) or aminophylline $(2.3 \mathrm{mg} / \mathrm{kg})$ every day during weeks 9-20. Normal saline was also orally administrated to control rats. On week 20, all rats were anesthetized and sacrificed to obtain lung tissues, blood, and bronchoalveolar lavage fluid. All experimental procedures were approved by the Experimental Animal Care and Ethics Committee of the First Affiliated Hospital, Henan University of Chinese Medicine. The medium BYF dose (4.4 g/ $\mathrm{kg}$ ) was calculated using the formula $\mathrm{D}$ rat=D human $\times$ (I rat/I human $) \times(\mathrm{W}$ rat $/ \mathrm{W}$ human $) 2 / 3$, where $\mathrm{D}=$ dose, $\mathrm{I}=$ body shape index, and $\mathrm{W}=$ body weight.

\section{Pulmonary Function and Histological Analyses}

To assess pulmonary function, tidal volume (TV), peak expiratory flow (PEF), and maximum minute ventilation (MMV) were measured using unrestrained plethysmography (Buxco Inc., Wilmington, NC, USA) every four weeks during weeks 0-20.

The left lower lobe was fixed with $10 \%$ formalin neutral buffer solution, embedded in paraffin, cut into $4-\mu \mathrm{m}$ sections, and stained with Mayer's hematoxylin and 1\% eosin alcohol solution (H\&E staining).

\section{Cytokine ELISA Assays}

IL-1 $\beta$, TNF- $\alpha$, IL-6, IL-17A, and IL-10 serum levels in the rats were measured with ELISA kits according to the manufacturer's instructions. All determinations were performed in triplicate.

\section{Flow Cytometry}

Cell suspensions containing $10^{6}$ cells $/ \mathrm{ml}$ were prepared. Cells were stained with FITC-anti-CD4 and APC-anti-CD25 antibodies, fixed, permeabilized with $0.1 \%$ Triton, and stained with PE-anti-Foxp3 and PE-anti-IL-17A antibodies. Isotype controls were included in all experiments to adjust the background signal. $\mathrm{T}$ cells were analyzed using a FACS CantoTM II (BD Biosciences, San Jose, CA, USA), and the results were analyzed with FlowJo7.6.1 software (Tree Star, USA).

\section{Quantitative Real-Time PCR}

Total RNA was extracted from spleens or cultured cells using Trizol according to the manufacturer's instructions (Invitrogen Corp.). Subsequently, RNA was reversed transcribed into cDNA using HiScript II Reverse Transcriptase (Vazyme, Nanjing, China). Real-time quantitative PCR was performed using a real-time reverse transcription (RT)-PCR (Applied Biosystems, CA, USA) based on general fluorescence detection by SYBR Green. For mRNA transcript analysis, gene-specific values were normalized to the Actb gene.

\section{Western Blot Assay}

Proteins were extracted from spleens, lung or cultured cells lysed with RIPA lysis buffer (Solarbio life sciences, Beijing, China) on ice and centrifuged, and then supernatants were collected. Total protein was mixed with SDS sample buffer, boiled, separated by SDS-PAGE, and transferred to PVDF membranes, which were blocked with non-fat milk for $2 \mathrm{~h}$. PVDF membranes were incubated overnight at $4^{\circ} \mathrm{C}$ with specific primary antibodies. After being washed three times with buffer, the membranes were incubated with HRP-conjugated secondary antibodies for $2 \mathrm{~h}$. Finally, the bands were visualized by film exposure with an ECL reagent.

\section{Statistical Analysis}

All values were expressed as means \pm standard errors of the means (S.E.M.). Statistical differences were assessed by one-way analysis of variance (ANOVA), followed by Tukey's post hoc test. Values of $\mathrm{P}<$ 0.05 were considered to indicate significant differences.

\section{RESULTS}

\section{Effect of BYF on Th17 and Treg Cell Differentiation In Vitro}

To explore how BYF restores the Th17/Treg balance, we first investigated the biological effect of BYF on Th17 cell differentiation. Naïve CD ${ }^{+} \mathrm{T}$ cells were exposed to anti-CD3, anti-CD28, TGF- $\beta$, and IL- 6 to promote their differentiation into Th17 cells in the presence of BYF. IL- 6 and TGF- $\beta$ cotreatment activates ROR $\gamma$ t, which is the critical transcription factor driving Th17 differentiation (Kimura and Kishimoto, 2010). BYF obviously suppressed ROR $\gamma$ t and IL-17A mRNA expression and ROR $\gamma$ t protein levels, suggesting that BYF inhibited Th17 cell differentiation (Figure 2). To investigate whether A2aR contributes to the BYF-mediated inhibition of Th17 cell differentiation, naïve CD4+ $\mathrm{T}$ cells were exposed to Th17stimulatory factors and KW6002 (A2aR antagonist) in the presence of BYF. The results showed that KW6002 significantly suppressed the inhibitory effect of BYF on ROR $\gamma t$ and IL-17 expression.

Treg cells exhibit immunosuppressive effects by expressing anti-inflammatory cytokines, such as IL-10. Additionally, TGF $\beta$ 

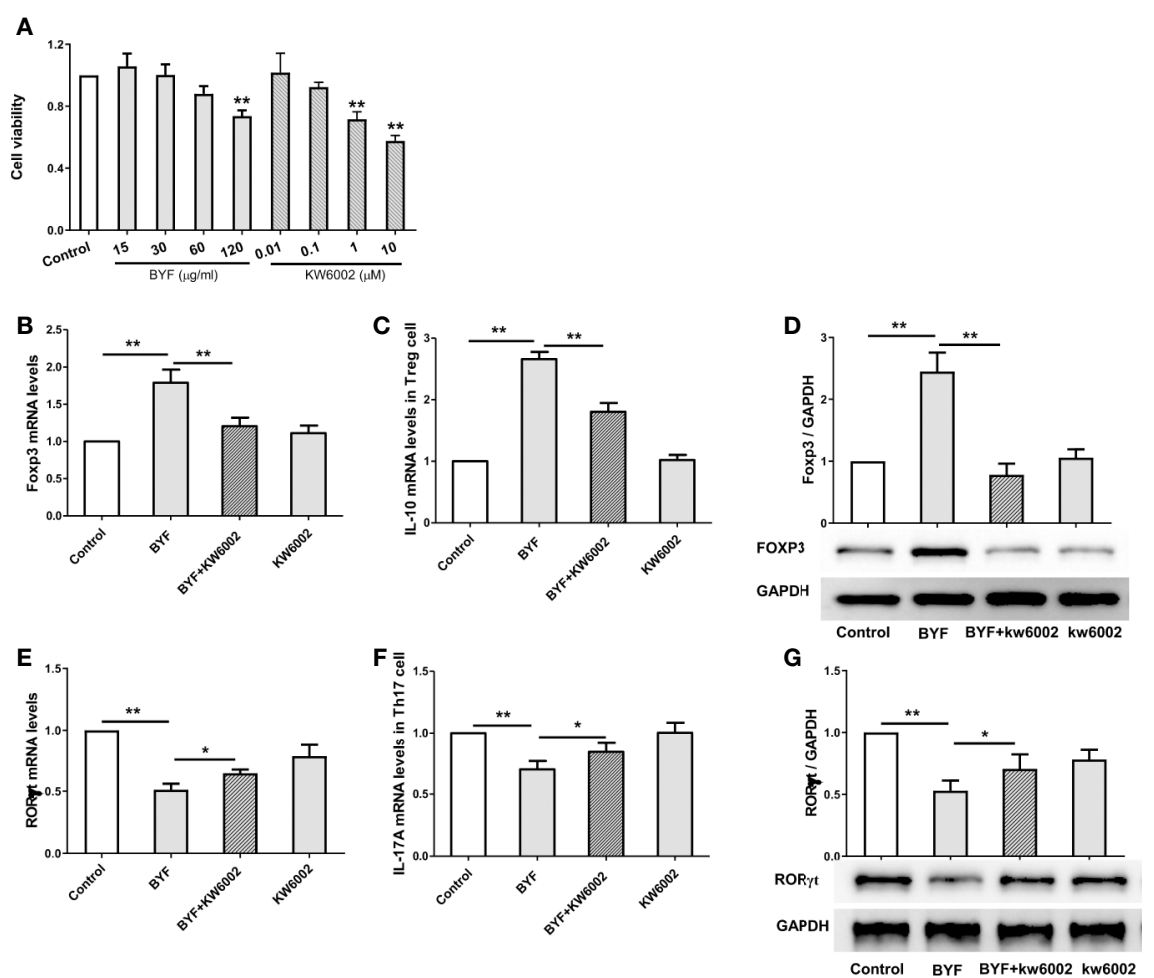

FIGURE 2 | Effect of Bufei Yishen formula (BYF) on Th17 and Treg cell differentiation. Naïve CD4+ T cells were isolated from spleens of mice, and treated with Th17 differentiation conditions or Treg differentiation conditions in the presence or absence of BYF (60 $\mu \mathrm{g} / \mathrm{ml})$ or KW6002 $(0.1 \mu \mathrm{M})$ for 3 days. (A) the effect of BYF and KW6002 on cell viability of Naiive CD4+ T cells. (B) The mRNA levels for Foxp3. (C) The mRNA levels IL-10. (D) The protein level of Foxp3. (E) RORYt mRNA; (F) IL17 mRNA; (G) RORyt protein. ${ }^{*} \mathrm{P}<0.05,{ }^{* *} \mathrm{P}<0.01$.

can selectively induce $\mathrm{T}$ cell differentiation into Tregs, which express higher Foxp3 levels (Kimura and Kishimoto, 2010). To further explore the effect of BYF on Treg cell differentiation, naïve CD4+ T cells were induced with anti-CD3, anti-CD28, and TGF- $\beta$ in the presence of BYF. BYF markedly increased the expression of the Treg markers Foxp3 and IL-10. Additionally, KW6002 obviously reduced the BYF-mediated increase in IL-10 and Foxp3 levels. These data demonstrated that BYF restored the Th17/Treg balance by regulating Th17 and Treg differentiation, in which A2aR might play an important role.

\section{Effect of BYF on A2aR Expression in Th17 and Treg Cells}

Next, we investigated the effect of BYF on A2aR expression during Th17 and Treg differentiation. BYF significantly increased A2aR RNA and protein levels in Th17 and Treg cells, which were suppressed by KW6002 (Figure 3). These data suggested that BYF increased A2aR expression via a positive feedback mechanism.

Previously, we showed that BYF regulated STAT3 and STAT5 phosphorylation. STAT3 activation regulates IL-6-mediated ROR $\gamma$ t expression and IL-17 production. Activated STAT5 binds to the Foxp3 gene promoter to regulate Treg cell activity (Korn et al., 2009; Kimura and Kishimoto, 2010). Here, we found that BYF decreased the STAT3 phosphorylation level and increased STAT5 phosphorylation in Th17 and Treg cells, which were inhibited by KW6002 (Figures 3E-H).

\section{Effect of A2aR Antagonist on the BYF- Reduced Severity of COPD}

KW6002 and BYF were administered to the COPD rats to verify the relevance of $\mathrm{A} 2 \mathrm{aR}$ activation and amelioration of COPD by BYF. The results showed that BYF significantly reduced the severity of COPD. However, KW6002 diminished the therapeutic effect of BYF on COPD, as indicated by the lung injury scores, bronchiole wall thickness, small pulmonary vessels wall thickness, bronchiole stenosis, alveolar diameters, increase in alveolar number, and lung functions (TV, PEF, and MMV) (Figures 4A-J). Additionally, KW6002 significantly reduced the inhibitory effect of BYF on IL- $1 \beta$, TNF- $\alpha$, and IL- 6 levels (proinflammatory cytokines) and Th17-related IL-17, as well as the increase in Treg-related IL-10 (Figures 5A-G). These results suggested that $\mathrm{A} 2 \mathrm{aR}$ activation was critical for the antiCOPD effect of BYF.

\section{Effect of KW6002 on the BYF-Restored Th17/Treg Balance in COPD Rats}

To verify the BYF-mediated regulation of Th17 and Treg cells via $\mathrm{A} 2 \mathrm{aR}$ in COPD rats, we further explored the effect of KW6002 

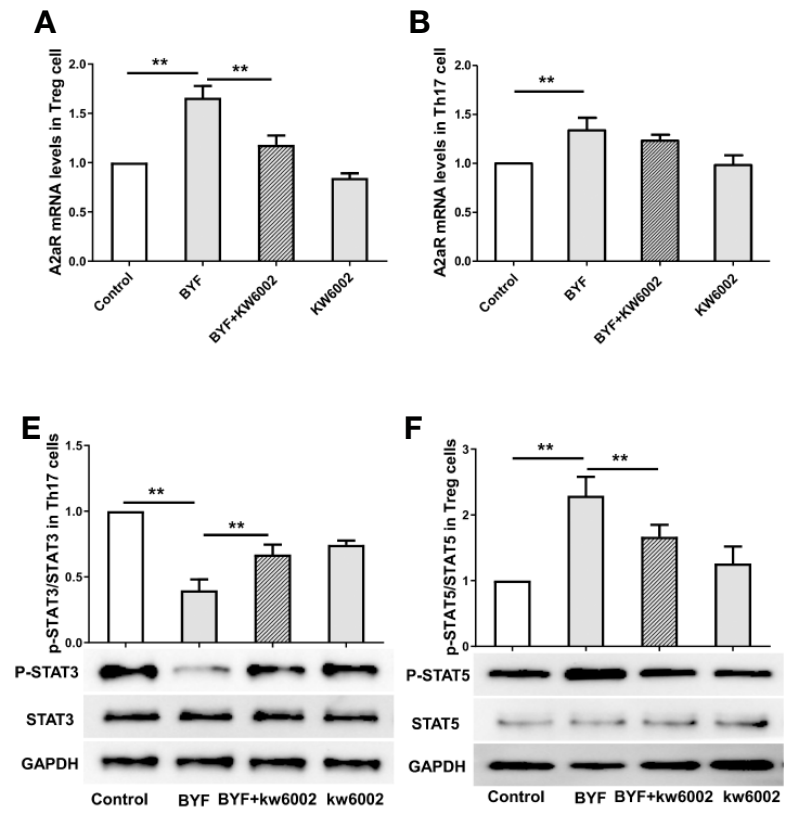

C

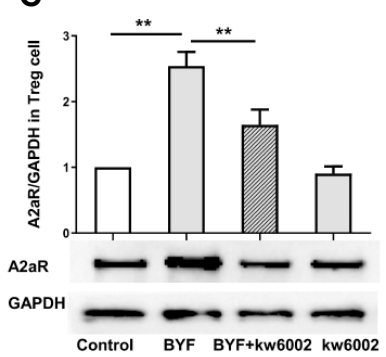

G

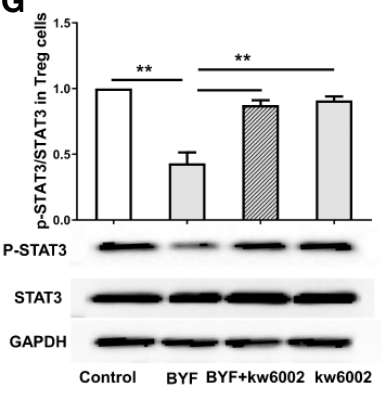

D

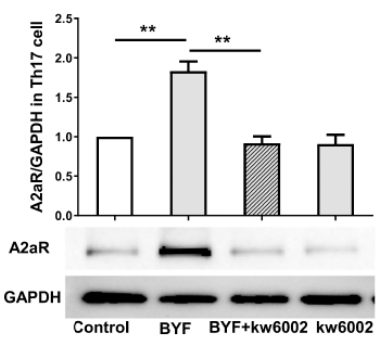

H

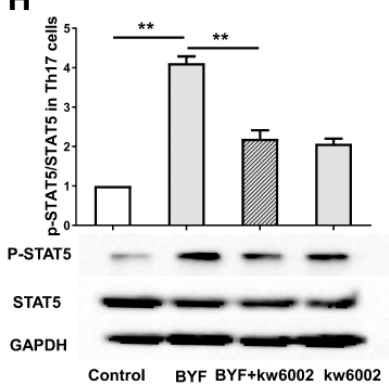

FIGURE 3 | Effect of Bufei Yishen formula (BYF) on A2aR and STAT3/5 in Th17 and Treg cells. Naiive CD4+ T cells were treated with Th17 differentiation conditions or Treg differentiation conditions in the presence or absence of BYF (60 $\mu \mathrm{g} / \mathrm{ml})$ or KW6002 $(0.1 \mu \mathrm{M})$ for 3 days. The mRNA levels of A2aR in Treg (A) and Th17 cells (B); the protein levels of A2aR in Treg (C) and Th17 cells (D); the protein levels of STAT3 and p-STAT3 in Th17 cells (E) and Treg cells (G) and STAT5 and p-STAT5 in Treg cells (F) and Th17 cells $(\mathbf{H}) .{ }^{* *} \mathrm{P}<0.01$.

on the BYF-restored Th17/Treg balance in rat spleens. BYF treatment obviously decreased the percentage of CD4+IL-17+ $\mathrm{T}$ cells and increased the percentage of CD4+CD25+Foxp3+ T cells (Figure 6). KW6002 reversed the decreased Th17 cell proportion and increased Treg cell proportion induced by BYF. KW6002 also reduced the inhibitory effect of BYF on ROR $\gamma$ t expression and its promotion of Foxp3 mRNA and protein expression (Figure 7). Additionally, BYF increased the $\mathrm{A} 2 \mathrm{aR}$ protein and mRNA levels in spleen tissues, and upregulated $\mathrm{A} 2 \mathrm{aR}, \mathrm{PKA}$ and $\mathrm{p}-\mathrm{CREB}$ proteins in lung tissues, which were inhibited by cotreatment with KW6002 (Figures 8A-C).

To further investigate whether A2aR signals contributes to the BYF-mediated differentiation of Th17 and Treg cells, we determined the effect of KW6002 on BYF-regulated STAT3 and STAT5 phosphorylation, the essential transcription factors for Th17 and Treg differentiation, in spleen tissues. We found that BYF treatment significantly reduced STAT3 phosphorylation and enhanced STAT5 phosphorylation, which were reversed by KW6002 cotreatment (Figures 8D-F). These results suggested that BYF regulated the Th17/Treg cell balance by activating $\mathrm{A} 2 \mathrm{aR}$ in $\mathrm{COPD}$ rats.

\section{DISCUSSION}

We previously showed that BYF treatment ameliorated lung function and pathological changes in COPD rats (Li et al., 2015;
Li Y. et al., 2016), which was mainly attributed to the inhibition of inflammatory responses and regulation of the Th17/Treg cell balance (Zhao et al., 2018). However, the mechanisms by which BYF mediates its anti-COPD effects remained unknown. In this study, we investigated how BYF regulates the Th17/Treg cell balance and COPD in rats. The data suggested that BYF inhibited Th17 differentiation and enhanced Treg differentiation by activating A2aR. Furthermore, A2aR antagonism suppressed the changes induced by BYF in Th17 cells, Treg cells, and COPD rats.

It is well established that Th17/Treg imbalance plays an important role in COPD development and progression (Yang et al., 2011). COPD patients exhibit increased Th17 cell populations accompanied by changes in their respective cytokines. Generally, Treg cells are functionally defective in COPD patients (Li X. N. et al., 2014). In this work, we found that BYF inhibited Th17 cell differentiation and enhanced Treg cell differentiation in vitro, which may contribute to the antiCOPD effect of BYF.

The exact mechanism by which BYF regulates Th17 and Treg differentiation is poorly understood. Many reports suggest that A2aR stimulation increases Treg cells and decreases Th17 subtypes (Li N. et al., 2012). Here, we found that BYF increased A2aR protein levels in Th17 and Treg cells, which were suppressed by KW6002. Additionally, STAT3 activated by IL-6, IL-23, and IL-21 enhanced ROR $\gamma$ t gene expression, the critical transcriptional factor for Th17 differentiation. STAT5 can directly bind to the Foxp3 gene to regulate the development 
A
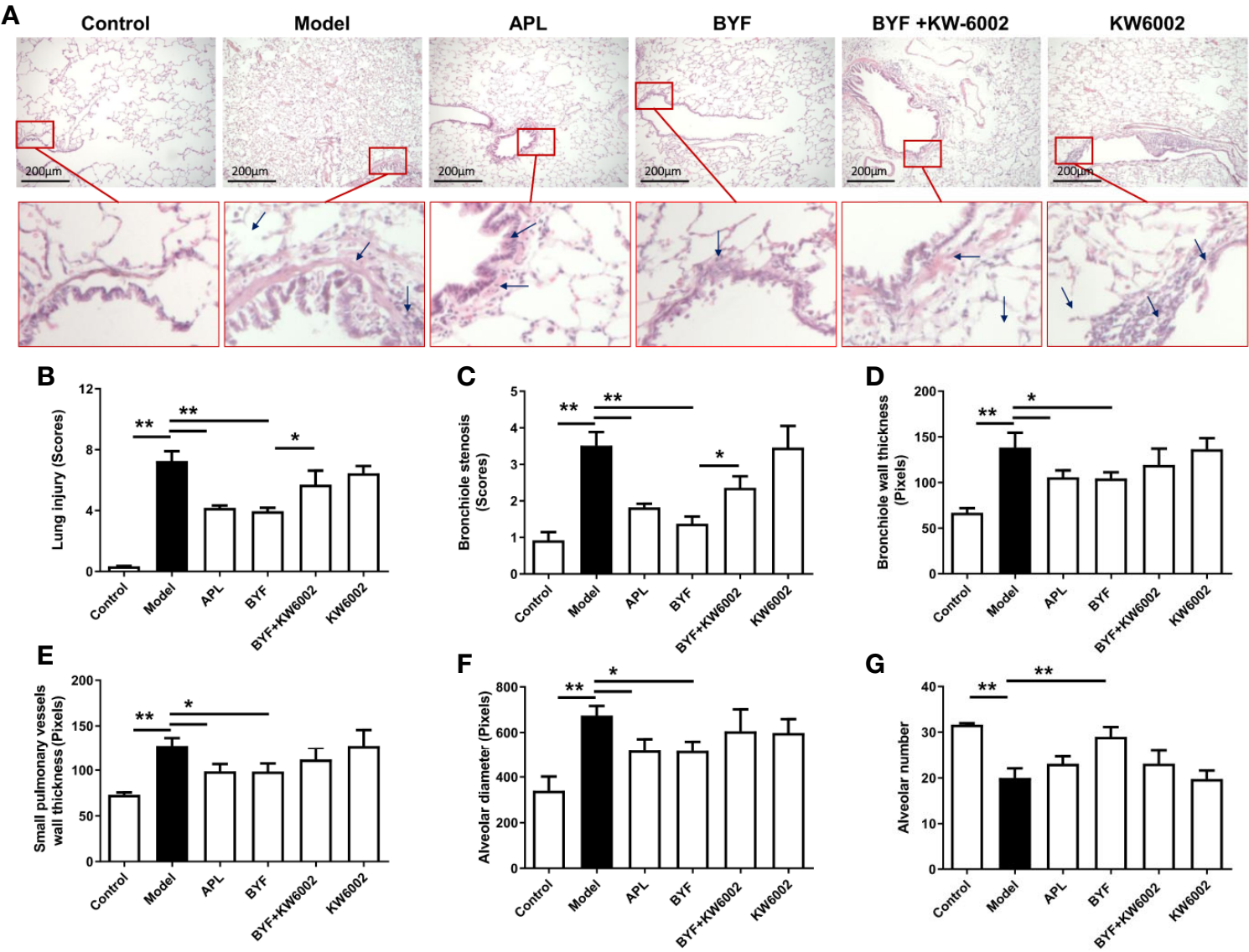

G
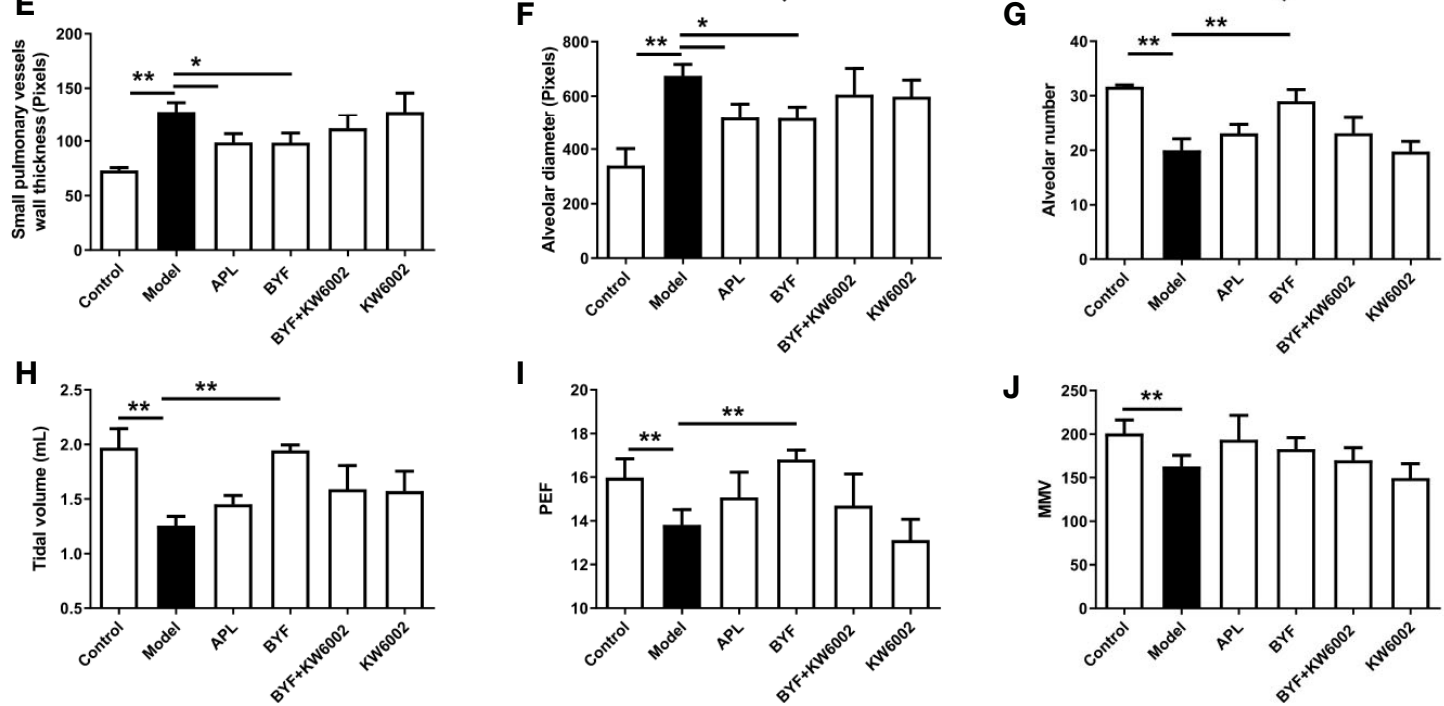

FIGURE 4 | Effect of cotreatment of Bufei Yishen formula (BYF) and A2aR antagonist on chronic obstructive pulmonary disease (COPD) in rats. COPD rats were orally administrated with BYF, KW6002 and aminophylline. (A) Histopathological changes of lung tissues of each group (HE staining, magnification, $\times 100)$. (B) Statistics of lung injury scores, (C) bronchiole stenosis, (D) bronchial wall thickness, (E) Small pulmonary vessels wall thickness, (F) alveolar diameter, (G) alveolar number, (H) Tidal volume (TV), (I) Peak expiratory flow (PEF), (J) Maximum minute ventilation (MMV). All data are presented as mean $\pm \mathrm{SEM}$. $\mathrm{N}=6$ for each group. ${ }^{*} \mathrm{P}<0.05,{ }^{* *} \mathrm{P}<0.01$

and maintenance of Treg cells. Here, we also showed that BYF increased the reduced STAT3 phosphorylation level and increased STAT5 phosphorylation in Treg and Th17 cells, which were reversed by KW6002 cotreatment.

A2aR-knockout mice show enhanced inflammation in their bronchial airways (Mohsenin et al., 2007). A2aR agonists may provide an alternative agent for the treatment of airway inflammatory diseases (Bonneau et al., 2006; Haskó et al., 2008; Golzar and Doukky, 2014). Thus, we examined the role of $\mathrm{A} 2 \mathrm{aR}$ in mediating the anti-COPD effect of BYF and its regulation of the Th17/Treg balance in COPD rats. The results showed that BYF could activate signaling of A2aR which leads to upregulation of PKA and CREB in lung tissues. Cotreatment with KW6002 diminished the therapeutic effect of BYF on COPD. Previously, regulation of the Th17/Treg balance was shown to play a key role in the anti-COPD effect of BYF (Zhao et al., 2018). In this work, we also demonstrated that KW6002 reversed the decrease in the Th17 cell proportion, the increase in the Treg proportion, and changes in STAT3 and STAT5 phosphorylation and A2aR signaling induced by BYF in COPD 

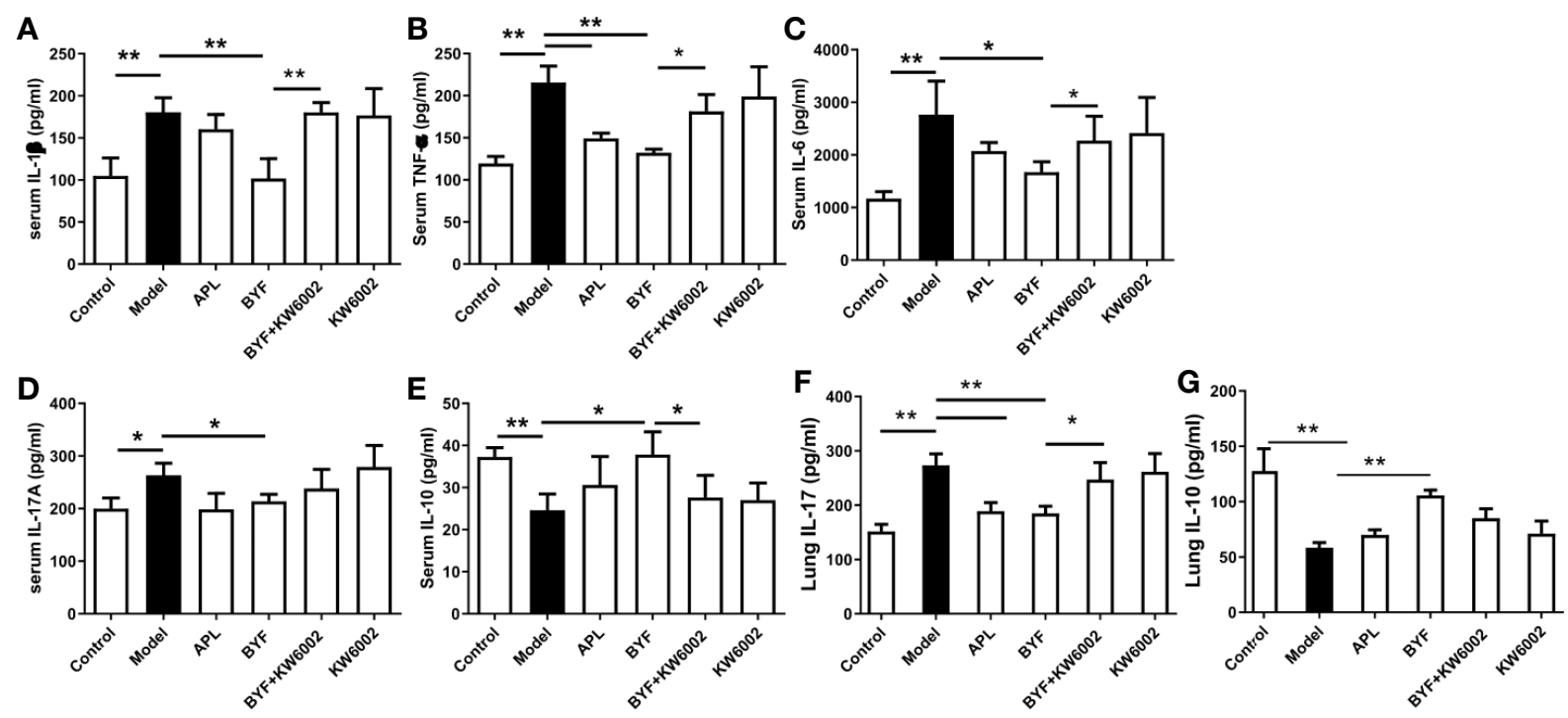

FIGURE 5 | Effect of cotreatment of Bufei Yishen formula (BYF) and A2aR antagonist on cytokine profiles in chronic obstructive pulmonary disease (COPD) rats. COPD rats were orally administrated with BYF, KW6002 and aminophylline. Cytokine levels of $f$ IL-1 $\beta$, TNF- $\alpha$, IL-6, IL-17A, and IL-10 in serum or lung tissues were detected by ELISA. The results were independently replicated. The values are presented as the means \pm SEM ( $n=6$ mice per group). ${ }^{\star} P<0.05,{ }^{* \star} P<0.01$.

A
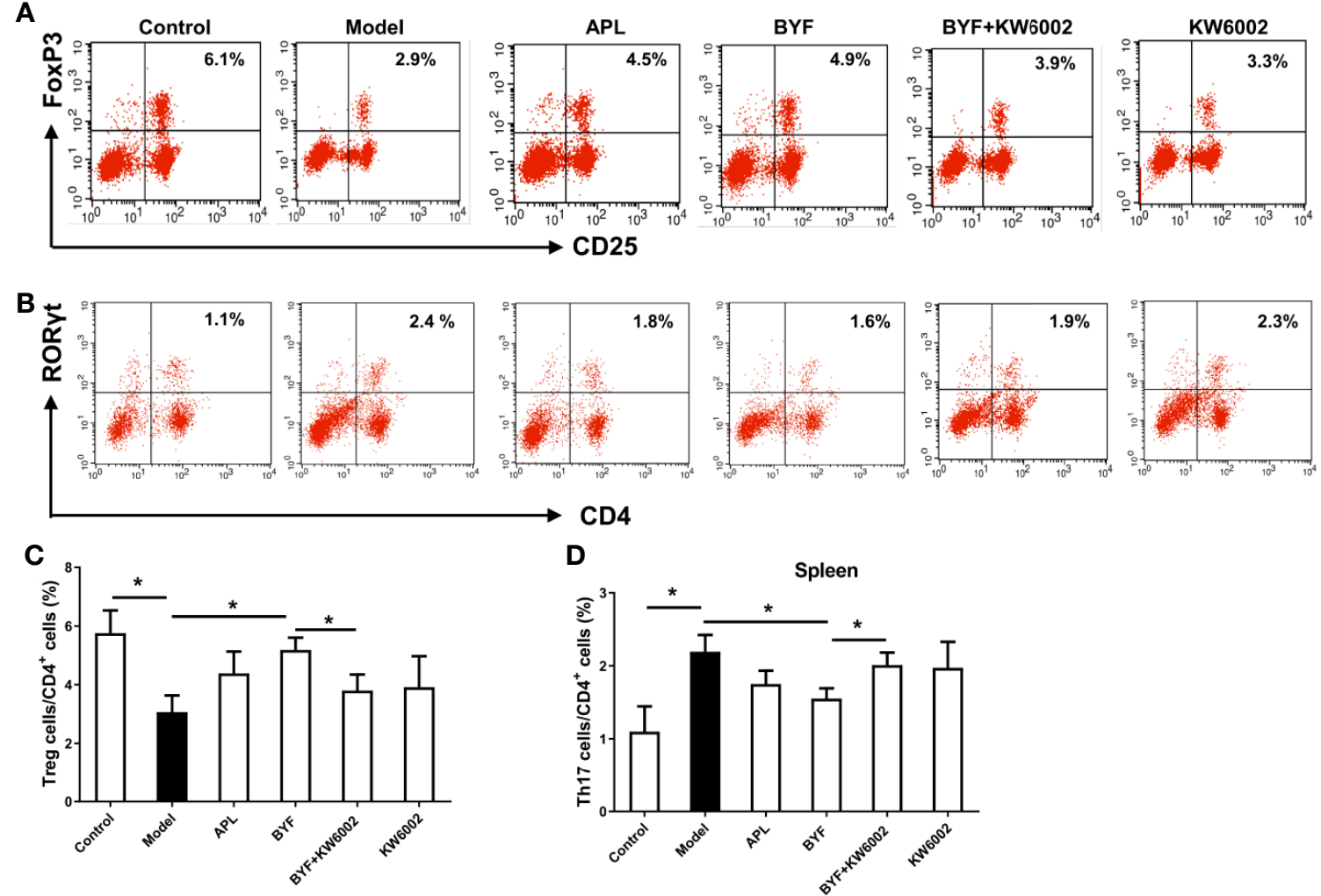

FIGURE 6 | Effect of cotreatment of Bufei Yishen formula (BYF) and A2aR antagonist on the balance of Th17/Treg cells in spleens of chronic obstructive pulmonary disease (COPD). COPD rats were orally administrated with BYF, KW6002 and aminophylline. The proportion of Treg (A) and Th17 cells (B) was detected. Statistics of the proportion of Treg (C) and Th17 cells (D) were analyzed. The values are presented as the means \pm SEM ( $n=6$ mice per group). ${ }^{*} P<0.05$. 
A
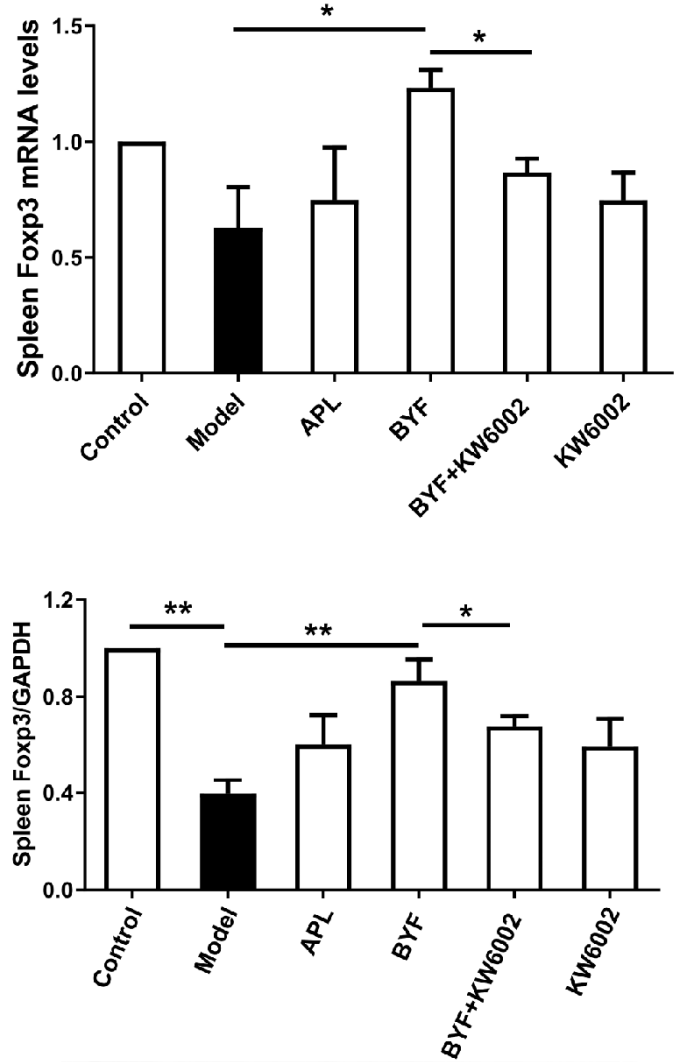

FOXP3

GAPDH

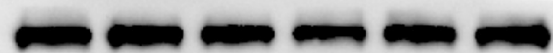

B
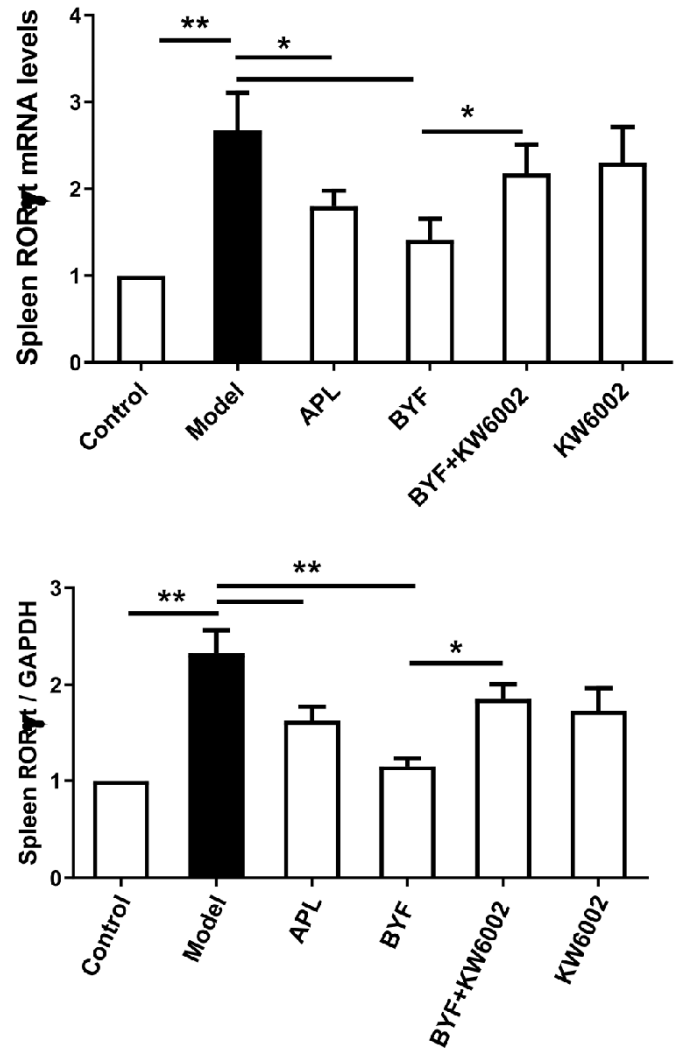

RORyt

GAPDH

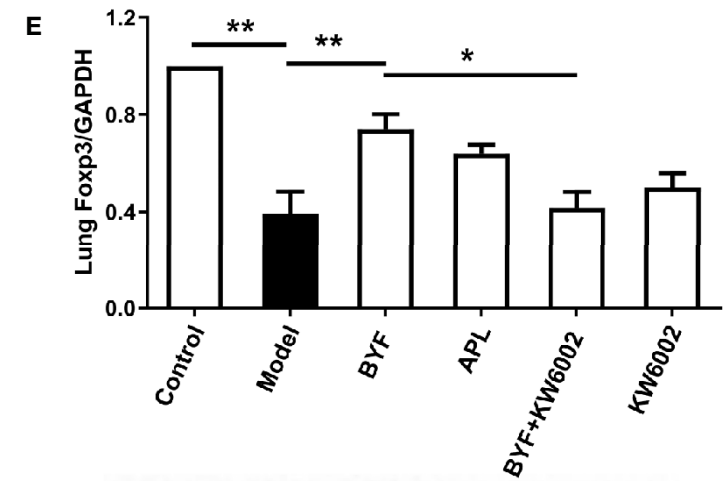

FOXP3

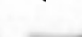

GAPDH

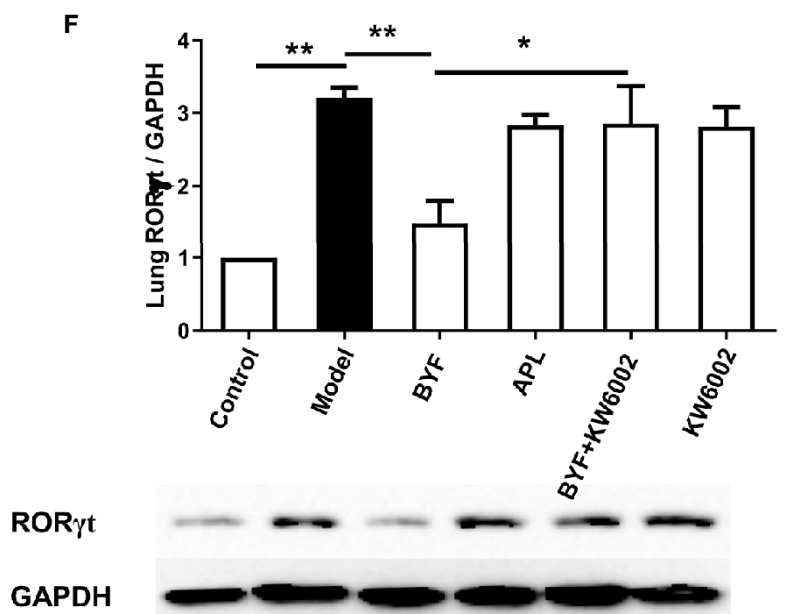

FIGURE 7 | Effect of cotreatment of Bufei Yishen formula (BYF) and A2aR antagonist on the mRNA levels of ROR pulmonary disease (COPD) rats. The mRNA levels for Foxp3 (A) and ROR $\gamma$ t (B) in the spleens were analyzed by a quantitative polymerase chain reaction (PCR)

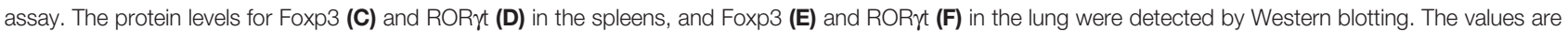
presented as the means \pm SEM ( $n=6$ mice per group). ${ }^{*} P<0.05$, ${ }^{* *} P<0.01$. 


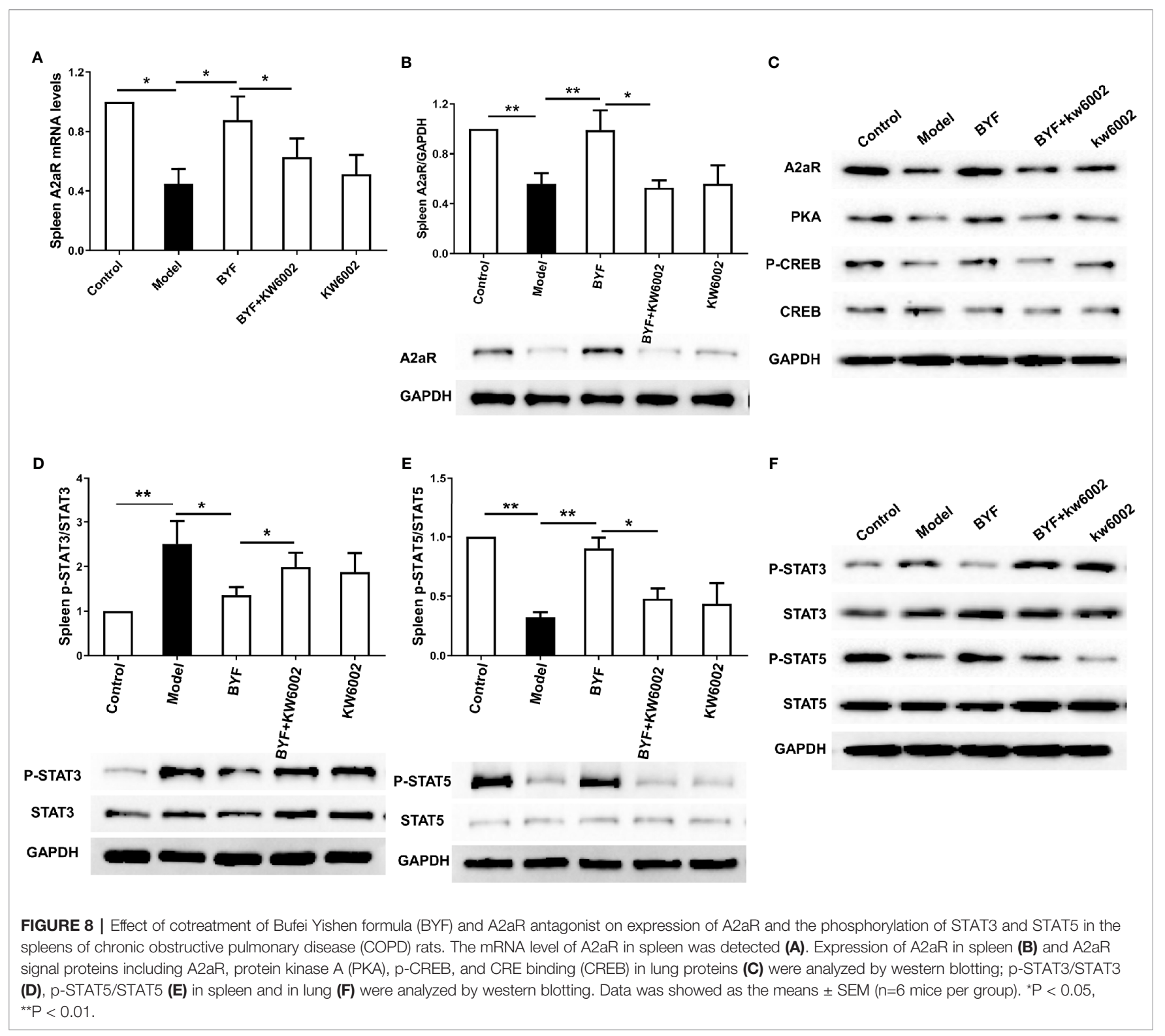

rats. These data demonstrate that activating A2aR plays a critical role in the therapeutic effect of BYF.

In conclusion, these results demonstrated that BYF exerted its anti-COPD efficacy by restoring the Th17/Treg balance via activating A2aR, which may help to elucidate the underlying immunomodulatory mechanism of BYF and provide evidence for its clinical application in COPD treatment.

\section{DATA AVAILABILITY STATEMENT}

The raw data supporting the conclusions of this article will be made available by the authors, without undue reservation, to any qualified researcher.

\section{ETHICS STATEMENT}

The animal study was reviewed and approved by Experimental Animal Care and Ethics Committee of the First Affiliated Hospital, Henan University of Chinese Medicine.

\section{AUTHOR CONTRIBUTIONS}

JL and PZ designed the outline of the study. PZ, XL, YT, and SF performed experiments, conceived the study, draft and revised the manuscript. DZ, ZR, XL, and HD were involved performing experiments, acquisition of data and statistical analysis. 
All authors contributed to the article and approved the submitted version.

\section{FUNDING}

The research is supported by National Natural Science Fund of China [grant number 81603473], Young Elite Scientists Sponsorship Program by CACM [grant number 2019-QNRC2B03], the Key Program of Henan Universities [grant number

\section{REFERENCES}

Barnes, P. J. (2016). Inflammatory mechanisms in patients with chronic obstructive pulmonary disease. J. Allergy Clin. Immunol. 138 (1), 16-27. doi: 10.1016/j.jaci.2016.05.011

Bonneau, O., Wyss, D., Ferretti, S., Blaydon, C., Christopher, S., and Stevenson, A. A. T. (2006). Effect of adenosine A2A receptor activation in murine models of respiratory disorders. Am. J. Physiol. Lung Cell Mol. Physiol. 290), L1036-L1043. doi: 10.1152/ ajplung.00422.2005

Brusselle, G. G., Joos, G. F., and Bracke, K. R. (2011). New insights into the immunology of chronic obstructive pulmonary disease. Lancet 378 (9795), 1015-1026. doi: 10.1016/S0140-6736(11)60988-4

Cervilha, D. A. B., Ito, J. T., Lourenço, J. D., Olivo, C. R., Saraiva-Romanholo, B. M., Volpini, R. A., et al. (2019). The Th17/Treg Cytokine Imbalance in Chronic Obstructive Pulmonary Disease Exacerbation in an Animal Model of Cigarette Smoke Exposure and Lipopolysaccharide Challenge Association. Sci. Rep. 9 (1). doi: 10.1038/s41598-019-38600-z

Eppert, B. L., Wortham, B. W., Flury, J. L., and Borchers, M. T. (2013). Functional characterization of $\mathrm{T}$ cell populations in a mouse model of chronic obstructive pulmonary disease. J. Immunol. 190 (3), 1331-1340. doi: 10.4049/ jimmunol.1202442

Golzar, Y., and Doukky, R. (2014). Regadenoson use in patients with chronic obstructive pulmonary disease: the state of current knowledge. Int. J. COPD 9), 129-137. doi: 10.2147/COPD.S56879

Haskó, G., Linden, J., Cronstein, B., and Pacher, P. (2008). Adenosine receptors: therapeutic aspects for inflammatory and immune diseases. Nat. Rev. Drug Discovery 7 (9), 759-770. doi: 10.1038/nrd2638

Jin, Y., Wan, Y., Chen, G., Chen, L., Zhang, M. Q., Deng, L., et al. (2014). Treg/IL17 ratio and Treg differentiation in patients with COPD. PloS One 9 (10), e111044. doi: 10.1371/journal.pone.0111044

Kimura, A., and Kishimoto, T. (2010). IL-6: Regulator of Treg/Th17 balance. Eur. J. Immunology 40 (7), 1830-1835. doi: 10.1002/eji.201040391

Korn, T., Bettelli, E., Oukka, M., and Kuchroo, A. V. K. (2009). IL-17 and Th17 Cells. Annu. Rev. Immunol. 27), 485-517. doi: 10.1146/annurev.immunol.021908.132710

Le Rouzic, O., Pichavant, M., Frealle, E., Guillon, A., Si-Tahar, M., and Gosset, P. (2017). Th17 cytokines: novel potential therapeutic targets for COPD pathogenesis and exacerbations. Eur. Respir. J. 50 (4), 1602434. doi: 10.1183/ 13993003.02434-2016

Li, N., Mu, L., Wang, J., Zhang, J., Xie, X., and Kong, Q. (2012). Activation of the adenosine A2A receptor attenuates experimental autoimmune myasthenia gravis severity. Eur. J. Immunol. 42), 1140-1151. doi: 10.1002/eji.201142088

Li, S., Li, J., Wang, M., Xie, Y., Yu, X. Q., Sun, Z. K., et al. (2012). Effects of comprehensive therapy based on traditional Chinese medicine patterns in stable chronic obstructive pulmonary disease: a four-center, open-label, randomized, controlled study. BMC Complement Altern. Med. 12:197. doi: 10.1186/1472-6882-12-197

Li, Y., Li, S. Y., Li, J. S., Deng, L., Tian, Y. G., Jiang, S. L., et al. (2012). A rat model for stable chronic obstructive pulmonary disease induced by cigarette smoke inhalation and repetitive bacterial infection. Biol. Pharm. Bull. 35 (10), 17521760. doi: $10.1248 / \mathrm{bpb} . b 12-00407$

Li, X. N., Pan, X., and Qiu, D. (2014). Imbalances of Th17 and Treg cells and their respective cytokines in COPD patients by disease stage. Int. J. Clin. Exp. Med. 7 (12), 5324-5329.
16A360004], and Project funded by China Postdoctoral Science Foundation [grant number 2016M602248].

\section{SUPPLEMENTARY MATERIAL}

The Supplementary Material for this article can be found online at: https://www.frontiersin.org/articles/10.3389/fphar.2020.01212/ full\#supplementary-material

Li, Y., Li, J. S., Li, W. W., Li, S. Y., Tian, Y. G., Lu, X. F., et al. (2014). Long-term effects of three Tiao-Bu Fei-Shen therapies on NF-kappaB/TGF-betal/smad2 signaling in rats with chronic obstructive pulmonary disease. BMC Complement Altern. Med. 14:140. doi: 10.1186/1472-6882-14-140

Li, J., Zhao, P., Li, Y., Tian, Y., and Wang, Y. (2015). Systems pharmacology-based dissection of mechanisms of Chinese medicinal formula Bufei Yishen as an effective treatment for chronic obstructive pulmonary disease. Sci. Rep. 5, 15290. doi: 10.1038/srep 15290

Li, J., Zhao, P., Yang, L., Li, Y., Tian, Y., and Li, S. (2016). System biology analysis of long-term effect and mechanism of Bufei Yishen on COPD revealed by system pharmacology and 3-omics profiling. Sci. Rep. 6 (1):25492. doi: 10.1038/ srep25492

Li, Y., Tian, Y., Li, J., Dong, Y., Wang, M., Feng, S., et al. (2016). Bufei Yishen granules combined with acupoint sticking therapy suppress oxidative stress in chronic obstructive pulmonary disease rats: Via regulating peroxisome proliferator-activated receptor-gamma signaling. J. Ethnopharmacology 193, 354-361. doi: 10.1016/j.jep.2016.08.027

Maigeng, Z., Haidong, W., Xinying, Z., Yin, P., and Jun, Z. (2019). Mortality, morbidity, and risk factors in China and its provinces 1990-2017: a systematic analysis for the Global Burden of Disease Study 2017. Lancet 394), 1145-1158. doi: 10.1016/S0140-6736(19)30427-1

Masjedi, A., Hassannia, H., Atyabi, F., Rastegari, A., Hojjat-Farsangi, M., Namdar, A., et al. (2019). Downregulation of A2AR by siRNA loaded PEG-chitosanlactate nanoparticles restores the $\mathrm{T}$ cell mediated anti-tumor responses through blockage of PKA/CREB signaling pathway. Int. J. Biol. Macromol. 133, 436445. doi: 10.1016/j.ijbiomac.2019.03.223

Mohsenin, A., Mi, T., Xia, Y., Kellems, R. E., Chen, J., and Blackburn, M. R. (2007). Genetic removal of the A2A adenosine receptor enhances pulmonary inflammation, mucin production, and angiogenesis in adenosine deaminasedeficient mice. Am. J. Physiol. Lung Cell Mol. Physiol. 293), L753-L761. doi: 10.1152/ajplung.00187.2007

Sales, D. S., Ito, J. T., Zanchetta, I. A., Annoni, R., Aun, M. V., Ferraz, L. F. S., et al. (2017). Regulatory T-Cell Distribution within Lung Compartments in COPD. COPD: J. Chronic Obstruct. Pulmon. Dis. 14 (5), 533-542. doi: 10.1080/ 15412555.2017.1346069

Trevethick, M., Mantell, S., Stuart, E., Barnard, A., Wright, K., and Yeadon, M. (2008). Treating lung inflammation with agonists of the adenosine A2A receptor: promises, problems and potential solutions. Br. J. Pharmacology 155), 463-474. doi: 10.1038/bjp.2008.329

Wang, C., Xu, J., Yang, L., Xu, Y., Zhang, X., Bai, C., et al. (2018). Prevalence and risk factors of chronic obstructive pulmonary disease in China (the China Pulmonary Health [CPH] study): a national cross-sectional study. Lancet 391 (10131), 1706-1717. doi: 10.1016/S0140-6736(18)30841-9

Yang, L., Ma, Q., Yao, W., Zhang, Q., Chen, H., Wang, G., et al. (2011). Relationship between the anti-inflammatory properties of salmeterol/ fluticasone and the expression of CD4+CD25+Foxp3+ regulatory $\mathrm{T}$ cells in COPD. Respir. Res. 12 (142), 1-11. doi: 10.1186/1465-9921-12-142

Yang, Y., Wang, H., Lv, X., Wang, Q., Zhao, H., Yang, F., et al. (2015). Involvement of cAMP-PKA pathway in adenosine $\mathrm{A} 1$ and $\mathrm{A} 2 \mathrm{~A}$ receptor-mediated regulation of acetaldehyde-induced activation of HSCs. Biochimie 115, 5970. doi: 10.1016/j.biochi.2015.04.019

Zarek, P. E., Huang, C., Lutz, E. R., Kowalski, J., Horton, M. R., Linden, J., et al. (2008). A2A receptor signaling promotes peripheral tolerance by inducing 
T-cell anergy and the generation of adaptive regulatory T cells. Blood 111), 251-259. doi: 10.1182/blood-2007

Zhang, F., Li, M., Lan, Y., and Wang, C. (2016). Imbalance of Th17/Tregs in rats with smoke inhalation-induced acute lung injury. Sci. Rep. 6 (1). doi: 10.1038/ srep21348

Zhao, P., Li, J., Tian, Y., Mao, J., and Liu, X. (2018). Restoring Th17/Treg balance via modulation of STAT3 and STAT5 activation contributes to the amelioration of chronic obstructive pulmonary disease by Bufei Yishen formula. J. Ethnopharmacol. 2018 (217), 152-162. doi: 10.1016/j.jep.2018. 02.023
Conflict of Interest: The authors declare that the research was conducted in the absence of any commercial or financial relationships that could be construed as a potential conflict of interest.

Copyright $\odot 2020$ Zhao, Liu, Dong, Tian, Feng, Zhao, Ren, Zhang and Li. This is an open-access article distributed under the terms of the Creative Commons Attribution License (CC BY). The use, distribution or reproduction in other forums is permitted, provided the original author(s) and the copyright owner(s) are credited and that the original publication in this journal is cited, in accordance with accepted academic practice. No use, distribution or reproduction is permitted which does not comply with these terms. 